\title{
Advances in wireless networks
}

\author{
Arjan Durresi $^{\mathrm{a}}$ and Mieso Denko ${ }^{\mathrm{b}}$ \\ ${ }^{\mathrm{a}}$ Department of Computer and Information Science at Indiana University-Purdue University \\ Indianapolis, 723 W. Michigan St., Indianapolis, IN 46202, USA \\ E-mail:durresi@cs.iupui.edu \\ ${ }^{\mathrm{b}}$ Department of Computing and Information Science, Guelph, ON, Canada, N1G 2W1 \\ E-mail:denko@cis.uoguelph.ca
}

\section{Introduction}

Wireless Networks, supported by recent technological advances in low power wireless communications along with silicon integration of various functionalities such as sensing, communications, intelligence and actuations are emerging as a critically important disruptive computer class based on a new platform, networking structure and interface that enable novel, low cost, high volume applications such as nuclear, biological and chemical attack detection and protection, home automation, battlefield surveillance and environmental monitoring. Several of such applications have been difficult to realize because of problems involved with inputting data from sensors directly in to actor systems. To fulfill their large range of applications wireless networks will collaborate with other wired networks. The research community is working to develop high performance computing solutions to problems arising from the complexities of these wireless network systems.

This special issue, highlights advances in various aspects of wireless networks and is organized mainly from the papers of the The 22nd IEEE International Conference on Advanced Information Networking and Applications AINA 2008, held in GinoWan, Okinawa, Japan, March 25-28, 2008. The conference received 469 submissions and every paper was reviewed carefully by 3 reviewers. Based on their quality and significance 146 papers were accepted in AINA 2008. We received 17 papers for this special issue. After two more rounds of review, we accepted 6 papers based on their quality and suitability to the special issue as well as the journal.

The Medium Access Control (MAC) protocol is a major element that determines the efficiency in sharing the limited communication bandwidth in wireless networks. In the first paper, Mjidi et al. study the impact of dynamic RTS threshold adjustment for IEEE 802.11 MAC protocol. They show both analytically and with extensive simulations the advantages of the proposed scheme.

In the second paper, Shigeyasu et al. propose a new MAC protocol, namely, IEEE802.11DCF with CCTS (Cancel CTS). The proposed protocol is analyzed by extensive simulations.

Research results on wireless ad hoc and sensor networks shows that most of the node energy is spent in idle state. Mahfoudh and Minet propose SERENA, an algorithm to SchEdule RoutEr Nodes Activity. 
SERENA is based on a two-hop coloring algorithm, whose complexity in terms of colors and rounds is evaluated. SERENA is compared with classical TDMA and optimized variants such as USAP in wireless ad hoc and sensor networks.

In the forth paper, Muhammad proposes an efficient method for introducing relay nodes in the given communication graph. The algorithm assigns transmitting ranges to the nodes such that the cost of range assignment function is minimal over all connecting range assignments in the graph. The output of the algorithm is the minimal Steiner tree on the graph consists of terminal (original) nodes and relay (additional) nodes.

Broadcasting is an essential and effective data propagation mechanism, with several of important applications such as route discovery, address resolution, as well as many other network services. In the next paper, Hanashi, Awan and Woodward propose a dynamic probabilistic approach that dynamically fine-tunes the rebroadcasting probability according to the number of neighbor's nodes distributed in the ad hoc network. The performance of the proposed approach is investigated and compared with the simple AODV and fixed probabilistic schemes using simulations under different mobility models.

In the last paper, Rohm et al. analyze via simulations the impact of different configurable MAC parameters on the performance of beaconless IEEE 802.15.4 networks under different traffic loads and under different levels of interference from the hidden nodes. Based on this analysis, we suggest the values of IEEE 802.15.4 MAC parameters that results in a good tradeoff between the packet loss probability and the packet latency under different conditions.

As we conclude this overview, we would like to thank all the authors for submitting their papers, and greatly thank many reviewers for their good work to make possible the publication of this special issue on time. In particular, we would like to address our special thanks to Dr. David Taniar for his strong encouragement and support to engage in this endeavor.

Arjan Durresi and Mieso Denko Guest Editors January, 2009

\begin{abstract}
Arjan Durresi received his B.E., MSc. and Ph.D. (all summa cum laude) in Electronic-Telecommunications, and a Diploma of Superior Specialization in Telecommunications from La Sapienza University in Rome, Italy and Italian Telecommunications Institute. Since August 2007, Dr. Durresi has been as an Associate Professor with the Department of Computer and Information Science, at Indiana University-Purdue University Indianapolis, IN, USA. Previously he has been with the Department of Computer Science at Louisiana State University, Department of Computer and Information Science in the Ohio State University, Telesoft S.p.A, Rome, Italy and Polytechnic University of Tirana. Dr. Durresi is a Professor of University in Albania and an Associate Member of Albania Academy of Science.

Dr. Durresi's current research interests include network architectures, heterogeneous wireless networks, security, QoS, routing protocols, traffic management, optical and satellite networks, multimedia networking, performance testing, and bioinformatics. Dr. Durresi has published more than seventy articles in journals and one hundred articles in proceedings of referred international conference. He is an area editor for the Ad Hoc Networks Journal (Elsevier), Journal of Network and Computer Applications(Elsevier), Journal of Ubiquitous Computing and Intelligence -JUCI, (American Scientific Publishers - ASP), and guest editor for the various International Journals. He is the founder of the IEEE International Workshops on Heterogeneous Wireless Networks - HWISE, and Co-Chair from 2005 to 2009. He is the Co-founder of the International Workshop on Advances in Information Security - WAIS and Co-Chair from 2007 to 2009. He is Co-Chair of AINA2009, Program Vice Chair of ICPADS 2005. Dr. Durresi is a Yamacraw Distinguished Speaker (2003) and a keynote speaker of IEEE AINA 2007. For more information, please visit www.cs.iupui.edu/ ${ }^{\sim}$ durresi.
\end{abstract}

Mieso Denko received his MSc degree from the University of Wales, UK, and his PhD degree from the University of Natal, South Africa, both in Computer Science. Since November 2002, Dr. Denko has been with the Department of Computing and 
Information Science, Guelph, Ontario, Canada, where he is now an Associate Professor with Tenure. His current research interests include wireless networks, mobile and pervasive computing, wireless mesh networks, wireless sensor networks and network security.

Dr. Denko is a founder/co-founder of a number of ongoing international workshops and served as program chair/co-chair of a number international conferences. Currently he is serving as guest co-editing of Special Issues for a number of journals including the ACM/Springer Mobile Networks and Applications (MONET) and IEEE Systems journal. Dr. Denko has coedited two books in the areas of pervasive computing and wireless networks, and currently co-editing a forthcoming book on autonomic computing and networking to be published by Springer in April 2009. He is Editorial board member of the Journal of Information Technology Research (JITR), IGI Publishing (2007-), International Journal of Smart Homes (IJSH), Editorial Advisory Board member of the Journal of Ubiquitous Computing and Communications, (UBICC), 2008-, and Associate Editor of the International Journal of Multimedia and Ubiquitous Engineering (IJMUE), SERSC (2008-), International Journal of Communication Systems (IJCS), Wiley, 2008- and Security and Communications Network (SCN), Wiley, 2009-. He is a senior member of the ACM and IEEE. For detailed information, please visit www.cis.uoguelph.ca/ ${ }^{\sim}$ denko. 

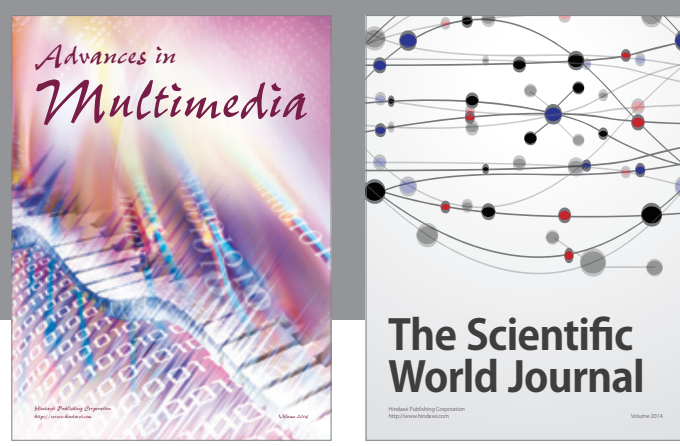

The Scientific World Journal
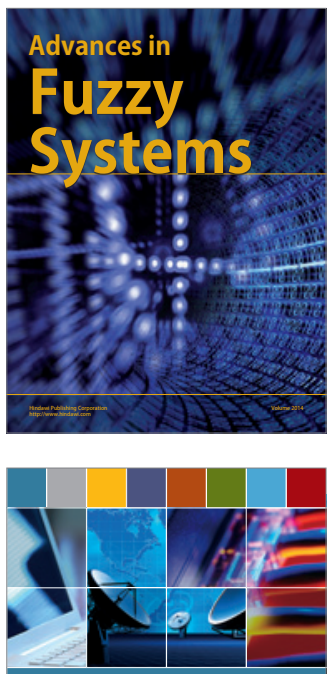

Computer Networks and Communications
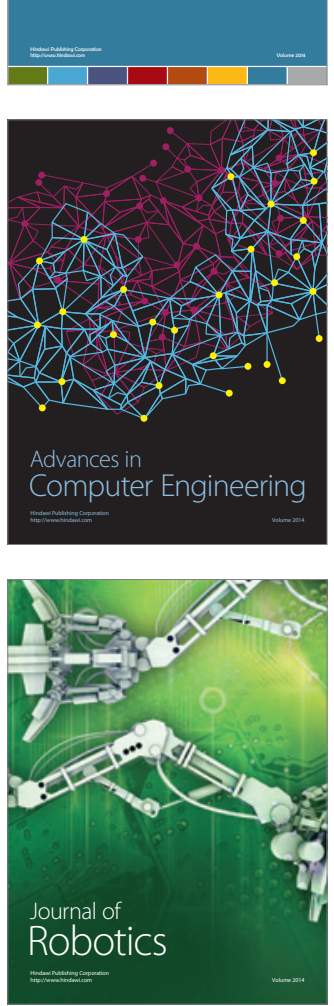
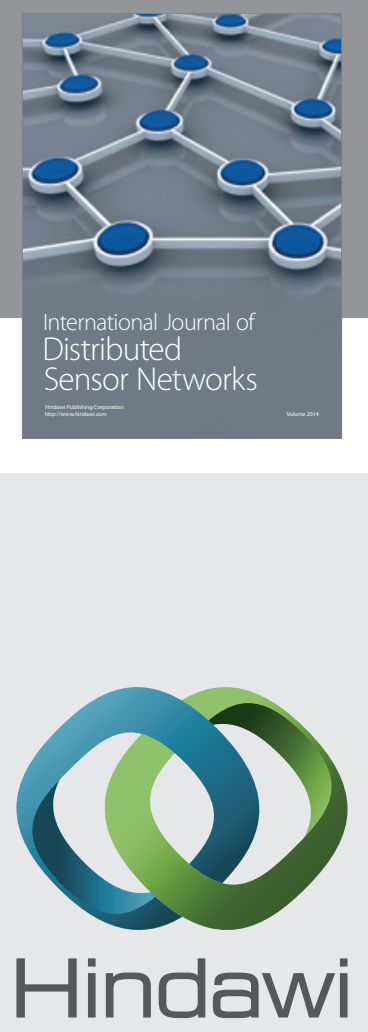

Submit your manuscripts at

http://www.hindawi.com
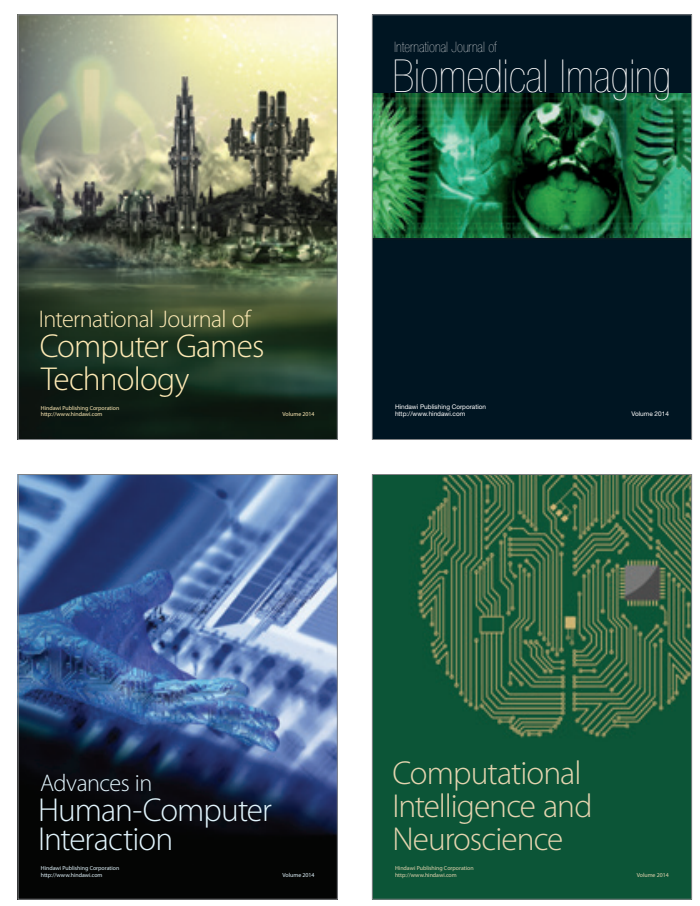
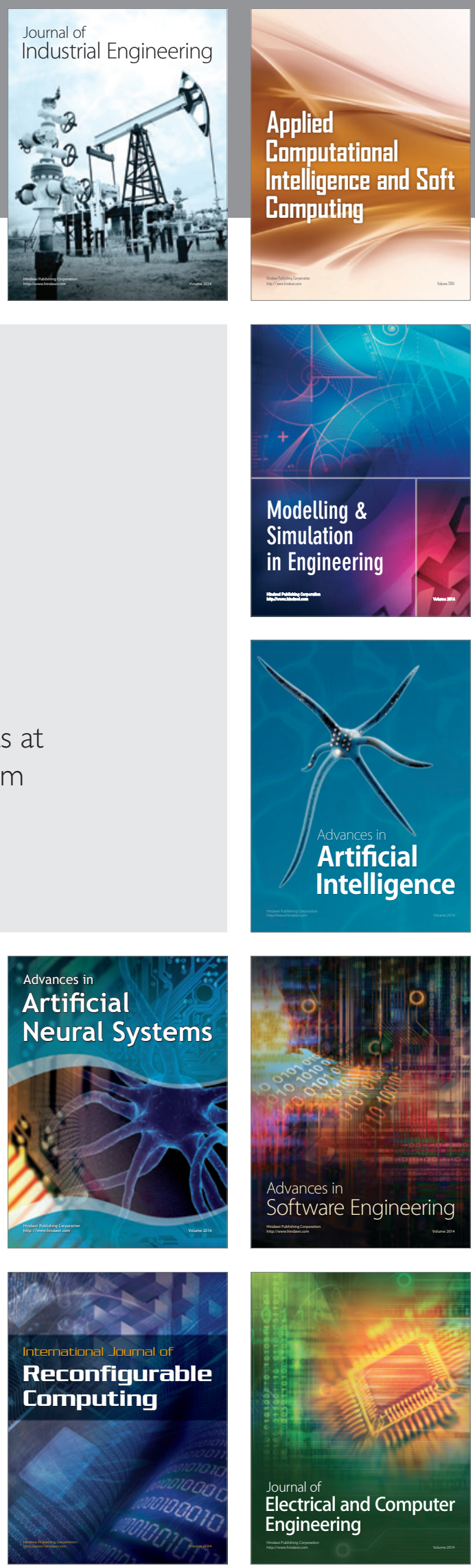